\title{
Existence and controllability results for second-order neutral stochastic equations with non-Lipschitz coefficient driven by Rosenblatt process
}

\author{
Abbes benchaabane ${ }^{1}$ and Ahmed Yahia Rakia ${ }^{2}$ \\ ${ }^{1}$ University of 8 May 1945 Guelma \\ ${ }^{2}$ University Centre Abdelhafid Boussouf Mila
}

May 23, 2020

\begin{abstract}
In this paper we consider a class of second-order impulsive stochastic functional differential equations driven simultaneously by a Rosenblatt process and standard Brownian motion in a Hilbert space. We prove an existence and uniqueness result under non-Lipschitz condition which is weaker than Lipschitz one and we establish some conditions ensuring the controllability for the mild solution by means of the Banach fixed point principle. At the end we provide a practical example in order to illustrate the viability of our result. \end } \{ \text { abstract } \}
\end{abstract}

\section{Hosted file}

Ahmed yahia-Benchaabane.pdf available at https://authorea.com/users/311192/articles/453557existence-and-controllability-results-for-second-order-neutral-stochastic-equations-

with-non-lipschitz-coefficient-driven-by-rosenblatt-process 